\title{
Mindfulness and Pain Catastrophizing on Chronic Disease Patients
}

\author{
Witrin Gamayanti ${ }^{1}$, A D Amalia ${ }^{2}$, Mursidin $^{3}$ \\ Department of Psychology, UIN Sunan Gunung Djati Bandung, Jl. A.H. Nasution No. 105, \\ Bandung 40614, Indonesia ${ }^{1,2,3}$ \\ \{witrin.gamayanti@uinsgd.ac.id $\left.{ }^{1}\right\}$
}

\begin{abstract}
So many patients experience chronic physical illness, some of them feel the pain excessively, which called pain catastrophizing. Pain catastrophizing causes the patient to feel excessive pain. It makes the patient become stress or depression. One that can relieve the pain catastrophizing is mindfulness. The aim of this research is to examine the correlation between mindfulness with pain catastrophizing in patients with chronic diseases. The participants were 40 patients with chronic disease who got treatment at the hospital. The instrument used in this research used Philadelphia Mindfulness Scale (PHLMS) and the Pain Catastrophizing Scale (PCS). The research used quantitative approach with analyses simple linear regression. The result shows that there is no significant relationship between mindfulness and pain catastrophizing.
\end{abstract}

Keyword : Mindfulness, Pain catastrophizing, Disease patients

\section{Introduction}

Catastrophizing pain (PC) is a psychological factor that can aggravate pain and contribute to influencing patients who are treated, this occurs because of the patient's perception of the pain he feels. With a PC, one can experience pain due to physical conditions, not in accordance with the actual conditions and this can worsen the patient's condition because the impact becomes hopeless and stressful [1]. A PC is a tendency to enlarge or overload camera values or pain without seriousness. Spanos (1979) emphasizes that pain will always be associated with feelings of worry and added, coupled with the inability to divert attention from pain [2]. The individual who owns a PC, his mind responds that he has pain and sees pain three times compared. As if he overestimated his thoughts about pain and pain [3]. PC is the most important among psychological factors that aggravate pain and contribute to chronicity of pain. PC is also a strong mediator of effects and pressure [4]. The study by Edwards et al. And Taylor et al. (2) stated that catastrophization of healthy vomiting behavior (health behavior) is beneficial for a person, such as exercise, which will aggravate symptoms [5]. According to Edwards et al. catastrophization on how to make patients pay more attention to their pain, increase pain perception and disappoint unpleasant [5]. Furthermore, 
catastrophization is high in fibromyalgia patients with greater activation in the brain area associated with emotion and modulation of pain money [5].

Mindfulness is defined as fact from one moment to another without judgment or judgment [6]. Research conducted by Nichole Elvery et al. [4] and Paul A. Frewen et al. [7] found that mindfulness training can reduce anxiety in people with heart disease. Elisabeth K.S (2013) proves that attention is very effective in reducing errors, depression, stress in a person. Anxiety, depression, and stress have characteristics and patterns similar to catastrophic pain. There is a significant positive between awareness with full awareness, non-judgmental (nonjudgmental) and not reactive in the sick victim [4]; positive channel between mindfulness and PC [8]; consciousness is negatively correlated with chest pain, PC, pain disorders, and depression, and these all positively correlate with mental health [9]. In Mindfulness there is an indicator of awareness needed to overcome negative thoughts and distress that often occur. Mindfulness is an activity that regulates mental development from mental alertness, focusing on oneself and enabling a better and more accurate environment, the patient will restore the psychological from negative thoughts and depression [10]. According to Prasetyo (2010) the factors that influence the feeling of one is the ability of his consciousness. The benefits of mindfulness are self-control [11], objectivity [12], long effects [13], increasing heart [12], inner balance (Morgan \& Morgan, 2005), emotional intelligence [14], and the ability to relate to others and yourself with kindness, acceptance, and compassion [13].

Previous studies have proven that mindfulness has information with PCs in people who experience physical pain. Research in Indonesia is still very minimal for a while in Indonesia, it is high enough to examine the relationship between mindfulness and PC in patients with chronic diseases.

\section{Methodology}

In this study quantitative methods with correlational methods using simple linear regression analysis. The population is 150 Internal Medicine Poly outpatients who are treated at a hospital in the city of Bandung, with a coverage period of 15-60 years. There were 63 patients who were willing to respond to the study respondents and 40 subjects who met the specified sample. The reliability test results of PCS measuring instruments with 14 items obtained a reliability coefficient of 0.766 which means high reliability. With PHLMS measuring instrument with 20 items Found a reliability coefficient of 0.756 which means high reliability too.

\section{Result and Discussion}

The results of statistical calculations in this study are presented in Table 1 and Table 2.

Table 1. Classical Assumption Test Results

\begin{tabular}{lcl}
\hline \multicolumn{1}{c}{ Test } & Score & \\
\hline Normality test & & \\
Mindfulness & 0.087 & Normal \\
Pain Catastrophizing & 0.461 & Normal \\
Homogenity test & & \\
Mindfulness & 0.477 & Homogen \\
Pain Catastrophizing & 0.796 & Homogen \\
\hline
\end{tabular}




\begin{tabular}{lll}
\hline Linierity test & & \\
$P C *$ Mindfulness & 0.493 & Linier \\
\hline
\end{tabular}

The results of inferential data analysis (table 2) regarding normality show that overall mindfulness with pain catastrophizing is normally distributed. For the Homogeneity Test, the analysis carried out in this study was a one-way ANOVA homogeneity test. The results show that both data are homogeneous (table 2). In the Linearity Test, the data are linear if a significant level is greater than 0.05 and the results of the data in this study prove linear data (table 2).

Table 2. The Results of Hypothesis with Regression Analyzes Test

\begin{tabular}{lll}
\hline \multicolumn{1}{c}{ Test } & \multicolumn{1}{c}{ Score } & \\
\hline Equality test correaltion & & No Correlate \\
$P C *$ Mindfulness & Sig 2 tailed $=0.208$ & \\
Regression test & & No effect \\
$P C *$ Mindfulness & $\mathrm{F}=1.639$ & \\
\hline
\end{tabular}

Table 3 presents the results of testing the hypothesis. Regression test produces a correlation coefficient of $r$ count $=0.203$ with a significance value of 0.208 , then with $n=40$ obtained $\mathrm{r}$ table with a 5\% error rate obtained $\mathrm{r}$ table 0.312 and with an error rate of $1 \%=$ 0.403. So $r$ count is smaller than $r$ table (Ho accepted) concluded that there is no significant relationship between mindfulness and pain catastrophizing. Then the correlation coefficient with a value of $0.203=$ a low level of correlation, means that the relationship between mindfulness and catastrophizing pain has a low level of correlation so the results of the hypothesis test reveal there is no relationship between the two. Anova test results show that mindfulness has no effect on catastrophizing pain in patients with chronic diseases. Next, information was obtained that patients with chronic diseases with high mindfulness and pain catastrophizing abilities were 13 people $32.5 \%$ ). Patients who have high mindfulness abilities and low catastrophizing pain are 7 people (17.5\%). Patients who have low mindfulness abilities but with high catastrophizing pain are 8 people $(20 \%)$. Patients who have low mindfulness abilities with low catastrophizing pain are 12 people $(30 \%)$.

Hypothesis test results show there is no correlation between mindfulness and catastrophizing pain (PC) in patients suffering from chronic diseases. The regression test also shows that mindfulness only provides an effective contribution of $4.1 \%$, the remaining $95.9 \%$ is influenced by other factors not examined in this study. The results of the categorization of cross distribution of mindfulness with PC have also given an idea that mindfulness does not have a significant relationship with PCs in patients with chronic disease. The results of cross distribution score categorization showed that 13 out of 40 patients with chronic disease had high mindfulness abilities with PCs and 12 of 40 people with chronic disease patients had low PC mindfulness.

This can happen because first, there are several factors that influence a person's perception of pain, especially chronic pain that they experience are as follows [15]: (a) age, (b) gender, (c) culture (d) meaning of pain, (e) location of pain, (f) attention or awarenesss, (g) anxiety, (h) fatigue (physiological conditions), (i) previous pain experience, and (j) family support. These factors can be measured in the scale of mindfulness PHLMS, while the remaining $97.9 \%$ cannot be measured by the scale of mindfulness used in this study such as age, gender, culture, anxiety, and family support. Then, related to the location of data retrieval factors, it requires calm (a supportive environment) during the measurement process so that the subject can be more mindful. Second, the subject routinely performs worship such as praying, 
recitation, holding a rosary, etc. to calm down and neutralize anxiety due to illness and pain. Also, sincerity and acceptance help reduce anxiety and pain. On the other hand, PHLMS measuring instruments only measure the ability of mindfulness towards themselves and their environment [16]. It can be said that PHLMS has not been able to measure and illustrate how individuals get calm and mindful abilities from the spiritual dimension. According to Sullivan [3], PHLMS is only able to reduce anxiety from the impact of the PC in the realm of the physical dimension magnify the pain (exaggerate pain) and ruminate the pain (thinking or imagining pain). In Islam, the concept of mindfulness is basically the same as that which Allah has commanded to know oneself, because by knowing yourself it will know its God [17], who knows himself and his god, his life will tend to be more mindful.

The results of this study are different from the results of research conducted by Nichole Elvery et al. [4], this difference according to researchers could be due to differences in the characteristics of the subject. In Nichole's research the subject was a student whose PC measured the most painful experience he had experienced.

\section{Conclusion}

The results of this study indicate that there is no significant relationship between mindfulness and PC, the correlation between the two is low and the Anova Test results also show that mindfulness has no effect on catastrophizing pain in patients with chronic diseases. $52.5 \%$ of subjects have high PCs, $47.5 \%$ have low PCs. For mindfulness, balanced high / low is $50 \%$. Coefficient of correlation with a value of $0.203=$ a low level of correlation so that the two variables have a low correlation.

\section{References}

[1] B. D. Darnall, J. A. Sturgeon, M. C. Kao, J. M. Hah, and S. C. Mackey, "From catastrophizing to recovery: A pilot study of a single-session treatment for pain catastrophizing," J. Pain Res., vol. 7, pp. 219-226, 2014.

[2] P. J. Quartana, C. M. Campbell, and R. R. Edwards, "Pain catastrophizing a critical review," Expert Review of Neurotherapeutics, vol. 9, no. 5. pp. 745-758, 2009.

[3] M. Sullivan, S. Bishop, and J. Pivik, "The pain catastrophizing scale: User Manual," Psychol. Assess., 2009.

[4] N. Elvery, M. P. Jensen, D. M. Ehde, and M. A. Day, "Pain Catastrophizing, Mindfulness, and Pain Acceptance," Clin. J. Pain, vol. 33, no. 6, pp. 485-495, 2017.

[5] K. Dorado, K. L. Schreiber, A. Koulouris, R. R. Edwards, V. Napadow, and A. Lazaridou, "Interactive effects of pain catastrophizing and mindfulness on pain intensity in women with fibromyalgia," Heal. Psychol. Open, vol. 5, no. 2, p. $205510291880740,2018$.

[6] D. M. Davis and J. A. Hayes, "What Are the Benefits of Mindfulness? A Practice Review of Psychotherapy-Related Research," Psychotherapy, vol. 48, no. 2. pp. 198 208, 2011.

[7] P. A. Frewen, E. M. Evans, N. Maraj, D. J. A. Dozois, and K. Partridge, "Letting go: Mindfulness and negative automatic thinking," Cognit. Ther. Res., vol. 32, no. 6, pp. 758-774, 2008.

[8] M. I. Jones and J. K. Parker, "Mindfulness mediates the relationship between mental 
toughness and pain catastrophizing in cyclists," Eur. J. Sport Sci., vol. 18, no. 6, pp. 872-881, 2018.

[9] P. Poulin et al., "The relationship between mindfulness, pain intensity, pain catastrophizing, depression, and quality of life among cancer survivors living with chronic neuropathic pain.," Support. Care Cancer, vol. 24, no. 10, pp. 4167-4175, 2016.

[10] S. C. Hayes and K. G. Wilson, "Mindfulness: Method and process," Clinical Psychology: Science and Practice, vol. 10, no. 2. pp. 161-165, 2003.

[11] S. R. et al. Bishop, "Mindfulness: A Proposed Operational Definition," Clin. Psychol. Sci. Pract., vol. 11, no. 3, p. 230, 2004.

[12] A. M. Hayes and G. Feldman, "Clarifying the construct of mindfulness in the context of emotion regulation and the process of change in therapy," Clin. Psychol. Sci. Pract., vol. 11, no. 3, pp. 255-262, 2004.

[13] P. R. Fulton, "Mindfulness as Clinical Training.," in Mindfulness and psychotherapy., 2005, pp. 55-72.

[14] R. Walsh and S. L. Shapiro, "The meeting of meditative disciplines and western psychology: A mutually enriching dialogue,” Am. Psychol., vol. 61, no. 3, pp. 227-239, 2006.

[15] L. D. Grant and B. E. Haverkamp, "A Cognitive-Behavioral Approach to Chronic Pain Management,” J. Couns. Dev., vol. 74, no. 1, pp. 25-32, 1995.

[16] L. Cardaciotto, J. D. Herbert, E. M. Forman, E. Moitra, and V. Farrow, "The assessment of present-moment awareness and acceptance: The philadelphia mindfulness scale," Assessment, vol. 15, no. 2, pp. 204-223, 2008.

[17] Al-Ghozali, Kimiya' Al Sa 'adah. Jakarta: Zaman, 2001. 